\title{
1 SodaPop: A Computational Suite for Simulating the Dynamics of Asexual Populations
}

2 page. cancer.

Louis Gauthier $^{1,2}$, Rémicia Di Franco ${ }^{1,2}$, Adrian W.R. Serohijos ${ }^{1,2}$

${ }^{1}$ Département de Biochimie, ${ }^{2}$ Centre Robert Cedergren en Bio-informatique et Génomique, Université de Montréal, 2900 Édouard-Montpetit, Montréal, Québec H3T 1J4, Canada

\section{Abstract}

Motivation: Simulating protein evolution with realistic constraints from population genetics is essential in addressing problems in molecular evolution, from understanding the forces shaping the evolutionary landscape to the clinical challenges of antibiotic resistance, viral evolution and

Results: To address this need, we present SodaPop, a new forward-time simulator of large asexual populations aimed at studying their structure, dynamics and the distribution of fitness effects with flexible assumptions on the fitness landscape. SodaPop integrates biochemical and biophysical properties in a cell-based, object-oriented framework and provides an efficient, open-source toolkit for performing large-scale simulations of protein evolution.

Availability and implementation: Source code and binaries are freely available at https://github.com/louisgt/SodaPop under the GNU GPLv3 license. The software is implemented in $\mathrm{C}++$ and supported on Linux, Mac OS/X and Windows.

25 
Introduction

29 Evolution predominantly depends on two causalities - population dynamics and the distribution 30 of fitness effects (DFE) (Eyre-Walker and Keightley, 2007). Despite the efforts to combine these 31 two causalities (DePristo et al., 2005; Silander et al., 2007; Goldstein, 2011; Liberles et al., 32 2012; Goldstein 2013; Serohijos and Shakhnovich, 2016; Echave and Wilke, 2017) there remains

33 a broad divide between population genetics and protein biophysics, both conceptually and 34 methodically. Bridging this gap is an essential step towards our understanding of molecular 35 evolution as a multi-scale process.

An important tool to study molecular evolution and compare outcomes of different

37 evolutionary scenarios is simulation. Methods to perform forward simulations vary in scope and

38 flexibility and are generally designed to investigate variety of problems in evolutionary biology

39 and population genetics such as polymorphism and population structure (Peng and Kimmel, 40 2005; Padhukasahasram et al., 2008; Hernandez, 2008; Carvajal-Rodriguez, 2008; O’Fallon,

41 2010; Thornton, 2014). These programs commonly implement features such as linkage and

42 recombination, specific migration, growth or mating schemes and selection regimes. Notably, 43 softwares such as OncoSimulR (Diaz-Uriarte, 2017) model the evolution of large asexual 44 populations, yet enforce strictly biallelic loci on limited sites. Likewise, there are several tools 45 intended to model protein evolution (Pang et al., 2005; Blackburne and Hirst, 2005; Koestler et 46 al., 2012; Grahnen and Liberles, 2012; Arenas et al., 2013). However, these programs are 47 typically aimed at phylogenetic reconstruction and alignment methods testing (Ziheng and 48 Rannala, 2012). Regardless of the practicality of current simulation packages in addressing problems in 50 human and population genetics, very few programs explicitly account for the DFE of proteins or 
51 integrate multiple scales in their evolutionary framework. Mutation and selection are indeed

52 separated by increasingly complex levels of biological organization. Studying molecular

53 evolution also requires accounting for higher-order scales such as systems and population.

54 Moreover, most molecular evolution simulators enforce a monoclonal regime, which does not

55 require the continuous tracking of an explicit population, but rather a single lineage. Despite the

56 higher computational tractability of this approach, evolution in large populations such as

57 bacterial colonies and malignant tumors is polyclonal, where the dynamics of segregating alleles

58 is of critical importance (Greaves and Maley, 2012; Lenski, 2017).

59 Here we introduce SodaPop, an efficient forward-time, object-oriented (OOP) simulator

60 aimed at studying the evolutionary dynamics of large-scale asexual populations with explicit

61 genomic sequences. In this framework, the population structure and the DFE of fixed mutations

62 can be explored simultaneously. Rather than being treated as a distribution (Haller and Messer,

63 2017; Kim et al., 2017), the DFE of arising mutations can be inputed from protein engineering

64 methods (Kumar et al., 2006; Yin et al., 2007; Laimer et al., 2015; Jia et al., 2015) or from

65 exhaustive mutagenesis experiments such as deep mutational scanning (Firnberg et al., 2014;

66 Fowler and Fields, 2014; Bloom, 2014). Also, SodaPop allows full flexibility in defining fitness

67 functions from biochemical/biophysical models that describe evolution of proteins. Additionally,

68 the OOP framework provides a scaffold where further developments can be easily integrated.

To our knowledge, SodaPop is the first publicly available and open source tool to this

70 end. The main program is implemented in $\mathrm{C}++$ as a command-line tool. We also provide

71 complementary tools to analyze and visualize simulation results. Source code, binaries and

72 documentation can be downloaded freely from https:/github.com/louisgt/SodaPop under the 
73 GNU GPLv3 license. Moreover, this software is portable on any POSIX-compliant operating

74 system, including Linux and Mac OS/X, or on Windows using the Cygwin environment.

75

76

\section{$77 \quad$ Methods}

78 SodaPop uses an adapted Wright-Fisher model with selection (Fisher, 1922; Wright, 1931).

79 Populations are characterized by a top-down organization: cells are dynamic objects comprising

80 a vector of genes, which are in turn defined by independent properties such as concentration or

81 abundance, functional essentiality and thermodynamic stability. Genetic sequences evolve

82 explicitly from one generation to the next, and can be traced back to the ancestral sequence

83 through an identifier. This hierarchical, object-oriented cell model marks a first step towards a

84 systems biology framework for the study of evolutionary dynamics. Generations are discrete

85 time steps in which each cell object gives birth to a number of children drawn from a binomial

86 distribution with mean equal to the fitness of the parent cell relative to the fitness summed over

87 all cells (Figure 1). These children form the basis for the next generation of cells. Following the

88 reproductive phase, the new population is scaled up or down to match the initial population size.

89 This process is akin to a serial passaging bottleneck experiments (Ebert, 1998; Gullberg et al., 90 2011).

The program can track all arising mutations during a simulation run to provide the full

92 history of genetic changes in the population. The program also tracks the associated selection 93 coefficients, which enables the temporal analysis of the DFE of substitutions. In addition,

94 SodaPop saves comprehensive snapshots of the population at a user-specified interval. This can

95 be tuned to an arbitrary granularity to yield an explicit genealogy of sequences and analyze the 
96 clonal dynamics. The population snapshots can also be used as input for subsequent simulations.

97 This feature facilitates the recovery of the latest state in a simulation in case of an unexpected

98 system crash. SodaPop is built upon streamlined data structures and a fast algorithm to achieve

99 high computational efficiency and to minimize the general trade-off between flexibility and 100 runtime (Carvajal-Rodriguez, 2008). The program is designed to support large population sizes

101 and rich substructures to reflect the natural magnitude of bacterial colonies and their intrinsic

102 dynamics. As such, SodaPop can readily handle simulations in the order of $10^{6}$ unique cells with

103 runtimes clocking under a few hours. We can reasonably tune the strength of selection or

104 mutation rate to achieve higher dynamical scales without incurring a significant computational

105 penalty.

106 SodaPop allows users to provide the nature and distribution of fitness effects as well as

107 the genotype-to-phenotype relationship to use in their simulation. The DFE of arising mutations

108 can be probabilistic, that is, defined by a distribution chosen by the user (Figure $2 \mathbf{A}$ ). It can also

109 be inputted from experiment (Figure 2B) or from computational estimates of biophysical

110 properties (Figure 2C). The ability to apply a specific fitness function based on input type

111 (Figure 2D) provides an additional layer of parameterization to the simulation. Altogether, these

112 capabilities establish a robust framework for the investigation of theoretical and applied

113 problems alike.

\section{Results}

116 In this section, we provide some examples of simulations performed by SodaPop. We present

117 simulations of protein evolution with different population genetics parameters as well as 118 different fitness functions. 


\section{Test case I: Population dynamics and fitness trajectories}

An evolutionary simulation with 10 genes in the folate biosynthesis pathway of

123 and incorporate additional protein properties such as catalytic efficiency or relative solvent

124 accessibility (see Supporting Information for details). One of the major aims of SodaPop is to

125 model rampant phenomena such as clonal interference and selective sweeps, which contribute

126 significantly to population dynamics (Elena and Lenski, 2003). The ability to investigate

127 polyclonal structure and relative fitness is of particular interest for co-culture competition assays

128 in microbiology (Lenski et al., 1998; Conrad et al., 2011; Melnyk and Kassen, 2011; Dragosits

129 and Mattanovich, 2013).

\section{Test case II: Multiple sequence alignment and conservation score}

132 To assess the performance of SodaPop in recapitulating the extent of amino acid conservation for

133 a protein that is under selection for stability, we compared simulated protein sequences to real

134 sequence data. It is known from in silico simulations that selection for protein folding stability

135 using physical force field estimations can reproduce the pattern of sequence conservation in real

136 biological sequences (Dokholyan and Shakhnovich, 2001; Ding and Dokholyan, 2006). To

137 construct ensemble of simulated "orthologs", we first primed a population by evolving it until it

138 reached a state of dynamic mutation-selection balance (Goyal et al., 2012). We then used the 139 output to perform 160 independent evolutionary simulations under selection for folding stability.

140 We ran each simulation for 700,000 generations, ensuring that the distribution of pairwise 141 sequence identities for simulated proteins matches that of the orthologs. For both sets, the 
142 distribution is a Gaussian centered around 36\% pairwise identity. Because each run produces as

143 many sequences as there are cells in the population, we narrowed down our set by randomly

144 sampling 5 sequences from each run for a total of 800 simulated DHFR sequences. For real

145 orthologous sequences, we retrieved the top 250 hits of a protein BLAST for the 192 amino acid

146 Candia albicans dihydrofolate reductase (DHFR), from which we excluded sequences longer

147 than $220 \mathrm{bp}$. We used the 163 remaining sequences to construct a multiple sequence alignment

148 using Clustal Omega (Sievers et al., 2011). To compare sequence conservation, we used the

149 Kullback-Leibler conservation score, which is a measure of relative entropy (Kullback and 150 Leibler 1951) for each residue $z$ :

$151 \quad K L_{z}=\sum_{i=1}^{N} P_{i} \ln \left(\frac{P_{i}}{Q_{i}}\right)$

(Equation 1)

152 where $P_{i}$ is the observed frequency of amino acid $i$ in that specific residue and $Q_{i}$ is the

153 background natural frequency of that specific amino acid shared amongst residues in orthologs.

154 A higher KL score implies a higher conservation of that residue's identity throughout evolution.

155 Conversely, when KL is closer to zero, that residue's identity is frequently substituted. Because

156 thermodynamic stability is the major evolutionary pressure on DHFR, our computational model

157 should be able to recapitulate the pattern of native sequence conservation. Indeed, as shown in

158 Figure 4, the sequence conservation of simulated DHFR sequences is significantly correlated

159 with real DHFR orthologs.

\section{Performance and runtime}

162 SodaPop is the first publicly available tool which can effectively simulate multi-scale molecular 163 evolution and polyclonal population dynamics in an all-encompassing framework. We 164 benchmarked SodaPop for multiple population sizes and number of generations. All simulations 
were run on a standard iMac desktop with a $3.2 \mathrm{GHz}$ Intel Core i5 processor and 16GB memory.

Figure 5 shows that runtime is quasi-monomial with respect to population size. We limited our

167 desktop benchmarking to $\mathrm{N}=10^{6}$ cells, as higher orders of magnitude induce a shift in

168 performance due to a RAM bottleneck. Explicit simulation of populations with higher orders of

169 magnitude requires a larger amount of memory than the current standard in commercial desktop

170 computers. Larger population sizes can be simulated on high-performance computing clusters

171 where memory allocation is not limiting. However, simulating up to a million cells for long time

172 periods is entirely tractable using standard desktop computers.

\section{Conclusion}

175 There are several features that can reduce the required memory for the performance of SodaPop.

176 First, using a binary encoding of the genetic code should reduce the memory required to store a

177 single cell by a significant factor without incurring any information loss. Second, collapsing 178 lineages within a single consensus sequence could also reduce the memory load, at a cost of 179 information loss. These are currently under development for future versions. Considering the 180 need to address questions at the interface of molecular evolution and population genetics, and

181 with most of the current computational methods unable to account for explicit clonal dynamics,

182 we believe SodaPop provides a comprehensive and extensible framework that can encompass a 183 wide array of evolutionary scenarios.

\section{Acknowledgements}

186 We would like to thank members of the Serohijos lab, especially Pouria Dasmeh, Ahn-Tien Ton

187 and Christopher Savoie for testing the program and contributing valuable questions and ideas. 


\section{Funding}

We acknowledge funds from the University of Montreal, NSERC, and the Merck Foundation.

Conflict of interest: none declared.

\section{References}

Arenas M, Dos Santos HG, Posada D, Bastolla U. Protein evolution along phylogenetic histories under structurally constrained substitution models. Bioinformatics. 2013 Dec $1 ; 29(23): 3020-8$.

Blackburne BP, Hirst JD. Population dynamics simulations of functional model proteins. J Chem Phys. 2005 Oct 15;123(15):154907.

Bloom JD. An experimentally determined evolutionary model dramatically improves phylogenetic fit. Mol Biol Evol. 2014 Aug;31(8):1956-78.

Carvajal-Rodríguez A. GENOMEPOP: a program to simulate genomes in populations. $B M C$ Bioinformatics. 2008 Apr 30; 9:223.

Carvajal-Rodríguez A. Simulation of genomes: a review. Curr Genomics. 2008 May;9(3):155-9. Conrad TM, Lewis NE, Palsson BØ. Microbial laboratory evolution in the era of genome-scale science. Mol Syst Biol. 2011 Jul 5; 7:509.

DePristo MA, Weinreich DM, Hartl DL. Missense meanderings in sequence space: a biophysical view of protein evolution. Nat Rev Genet. 2005 Sep;6(9):678-87.

Diaz-Uriarte R. OncoSimulR: genetic simulation with arbitrary epistasis and mutator genes in asexual populations. Bioinformatics. 2017 Feb 10.

Ding F, Dokholyan NV. Emergence of protein fold families through rational design. PLoS Comput Biol. 2006 Jul 7;2(7):e85.

Dokholyan NV, Shakhnovich EI. Understanding hierarchical protein evolution from first principles. J Mol Biol. 2001 Sep 7;312(1):289-307.

Dragosits M, Mattanovich D. Adaptive laboratory evolution -- principles and applications for biotechnology. Microb Cell Fact. 2013 Jul 1; 12:64.

Ebert D. Experimental evolution of parasites. Science. 1998 Nov 20;282(5393):1432-5.

Echave, Julian, and Claus O. Wilke. "Biophysical models of protein evolution: Understanding the patterns of evolutionary sequence divergence." Annual Review of Biophysics 46 (2017): 85-103.

Elena SF, Lenski RE. Evolution experiments with microorganisms: the dynamics and genetic bases of adaptation. Nat Rev Genet. 2003 Jun;4(6):457-69.

Eyre-Walker A, Keightley PD. The distribution of fitness effects of new mutations. Nature reviews. Genetics 8.8 (2007): 610.

Firnberg E, Labonte JW, Gray JJ, Ostermeier M. A comprehensive, high-resolution map of a gene's fitness landscape. Mol Biol Evol. 2014 Jun;31(6):1581-92. 
Fisher RA. On the dominance ratio. 1922. Bull Math Biol. 1990;52(1-2):297-318;

Fowler DM, Fields S. Deep mutational scanning: a new style of protein science. Nat Methods. 2014 Aug;11(8):801-7.

Geiler-Samerotte KA, Dion MF, Budnik BA, Wang SM, Hartl DL, Drummond DA. Misfolded proteins impose a dosage-dependent fitness cost and trigger a cytosolic unfolded protein response in yeast. Proc Natl Acad Sci U S A. 2011 Jan 11;108(2):680-5.

Goldstein RA. Population size dependence of fitness effect distribution and substitution rate probed by biophysical model of protein thermostability. Genome Biol Evol. 2013;5(9):1584-93.

Goldstein, RA. "The evolution and evolutionary consequences of marginal thermostability in proteins." Proteins: Structure, Function, and Bioinformatics 79.5 (2011): 1396-1407.

Goyal S, Balick DJ, Jerison ER, Neher RA, Shraiman BI, Desai MM. Dynamic mutationselection balance as an evolutionary attractor. Genetics. 2012 Aug;191(4):1309-19.

Greaves M, Maley CC. Clonal evolution in cancer. Nature. 2012 Jan 18;481(7381):306-13.

Gullberg E, Cao S, Berg OG, Ilbäck C, Sandegren L, Hughes D, Andersson DI. Selection of resistant bacteria at very low antibiotic concentrations. PLoS Pathog. 2011 Jul;7(7):e1002158.

Grahnen, J., Liberles, D. CASS: Protein sequence simulation with explicit genotype-phenotype mapping. Trends in Evolutionary Biology, 2012 4(1), e9.

Haller BC, Messer PW. SLiM 2: Flexible, Interactive Forward Genetic Simulations. Mol Biol Evol. 2017 Jan;34(1):230-240.

Hernandez RD. A flexible forward simulator for populations subject to selection and demography. Bioinformatics. 2008 Dec 1;24(23):2786-7.

Jia L, Yarlagadda R, Reed CC. Structure Based Thermostability Prediction Models for Protein Single Point Mutations with Machine Learning Tools. PLoS One. 2015 Sep 11;10(9):e0138022.

Kessner D, Novembre J. forqs: forward-in-time simulation of recombination, quantitative traits and selection. Bioinformatics. 2014 Feb 15;30(4):576-7.

Kim BY, Huber CD, Lohmueller KE. Inference of the Distribution of Selection Coefficients for New Nonsynonymous Mutations Using Large Samples. Genetics. 2017 May;206(1):345-361.

Koestler T, von Haeseler A, Ebersberger I. REvolver: modeling sequence evolution under domain constraints. Mol Biol Evol. 2012 Sep;29(9):2133-45.

Kullback S, Leibler RA. 1951. On information and sufficiency. Ann Math Stat 22:79-86.

Kumar MD, Bava KA, Gromiha MM, Prabakaran P, Kitajima K, Uedaira H, Sarai A. ProTherm and ProNIT: thermodynamic databases for proteins and protein-nucleic acid interactions. Nucleic Acids Res. 2006 Jan 1;34: D204-6.

Laimer J, Hofer H, Fritz M, Wegenkittl S, Lackner P. MAESTRO--multi agent stability prediction upon point mutations. BMC Bioinformatics. 2015 Apr 16; 16:116.

Lenski RE, Mongold JA, Sniegowski PD, Travisano M, Vasi F, Gerrish PJ, Schmidt TM. Evolution of competitive fitness in experimental populations of E. coli: what makes 
one genotype a better competitor than another? Antonie Van Leeuwenhoek. 1998 Jan;73(1):35-47.

Lenski RE. Experimental evolution and the dynamics of adaptation and genome evolution in microbial populations. ISME J. 2017 May 16.

Liberles, David A., et al. "The interface of protein structure, protein biophysics, and molecular evolution." Protein Science 21.6 (2012): 769-785.

Melnyk AH, Kassen R. Adaptive landscapes in evolving populations of Pseudomonas fluorescens. Evolution. 2011 Nov;65(11):3048-59.

O'Fallon B. TreesimJ: a flexible, forward time population genetic simulator. Bioinformatics. 2010 Sep 1;26(17):2200-1.

Padhukasahasram B, Marjoram P, Wall JD, Bustamante CD, Nordborg M. Exploring population genetic models with recombination using efficient forward-time simulations. Genetics. 2008 Apr;178(4):2417-27.

Pang A, Smith AD, Nuin PA, Tillier ER. SIMPROT: using an empirically determined indel distribution in simulations of protein evolution. BMC Bioinformatics. 2005 Sep 27; 6:236.

Peng B, Kimmel M. simuPOP: a forward-time population genetics simulation environment. Bioinformatics. 2005 Sep 15;21(18):3686-7.

Serohijos AW, Shakhnovich EI. Contribution of selection for protein folding stability in shaping the patterns of polymorphisms in coding regions. Mol Biol Evol. 2014 Jan;31(1):16576.

Serohijos AW, Shakhnovich EI. Merging molecular mechanism and evolution: theory and computation at the interface of biophysics and evolutionary population genetics. Curr Opin Struct Biol. 2014 Jun; 26:84-91.

Sievers F, Wilm A, Dineen D, Gibson TJ, Karplus K, Li W, Lopez R, McWilliam H, Remmert M, Söding J, Thompson JD, Higgins DG. Fast, scalable generation of high-quality protein multiple sequence alignments using Clustal Omega. Mol Syst Biol. 2011 Oct $11 ; 7: 539$.

Silander OK, Tenaillon O, Chao L. Understanding the evolutionary fate of finite populations: the dynamics of mutational effects. PLoS Biol. 2007 Apr;5(4):e94.

Tahmasbi R, Keller MC. GeneEvolve: a fast and memory efficient forward-time simulator of realistic whole-genome sequence and SNP data. Bioinformatics. 2017 Jan 15;33(2):294-296.

Thornton KR. A C++ template library for efficient forward-time population genetic simulation of large populations. Genetics. 2014 Sep;198(1):157-66.

Wright S. Evolution in Mendelian Populations. Genetics. 1931 Mar;16(2):97-159.

Yin S, Ding F, Dokholyan NV. Eris: an automated estimator of protein stability. Nat Methods. 2007 Jun;4(6):466-7.

Zanini F, Neher RA. FFPopSim: an efficient forward simulation package for the evolution of large populations. Bioinformatics. 2012 Dec 15;28(24):3332-3.

Ziheng Y, Rannala B. Molecular phylogenetics: principles and practice. Nature reviews. Genetics 13.5 (2012): 303. 


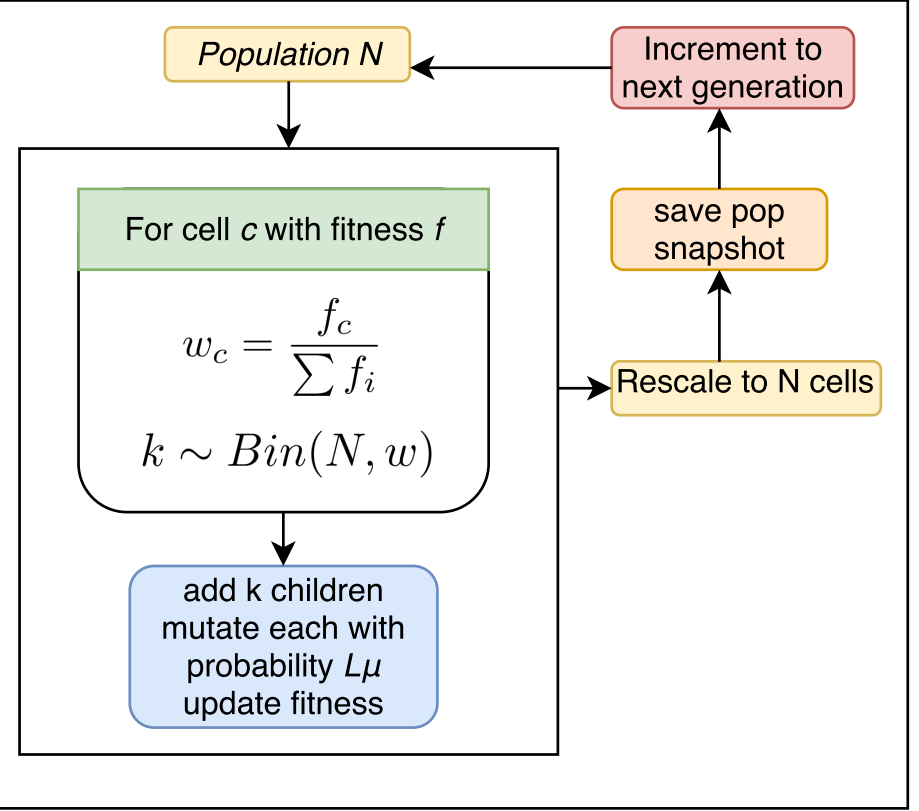

Figure 1. Illustration of SodaPop's core algorithm. The Wright-Fisher process iterates through every cell and draws the number of children to add to the next generation. These children are mutated with probability $L \mu$, where $L$ is the genomic length and $\mu$ is the mutation rate. Once the whole parent population has been swapped with daughter cells, this new generation is rescaled to $N$ cells.

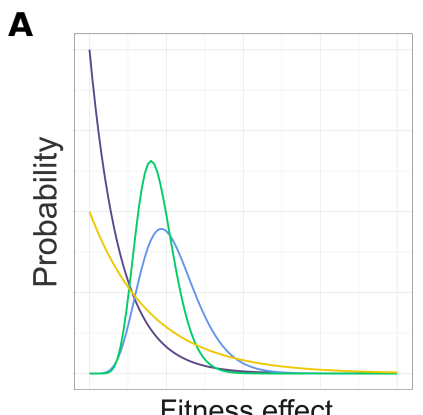

C

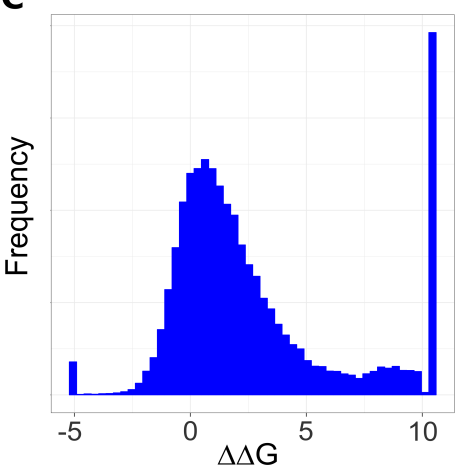

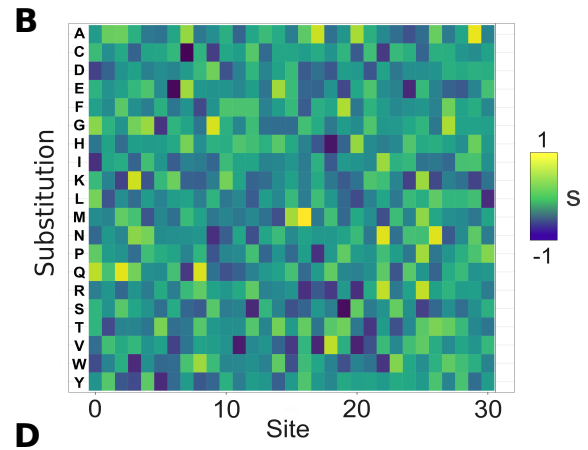

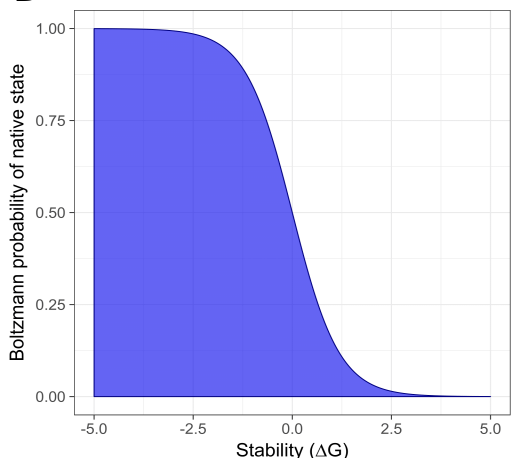

Figure 2. SodaPop accepts various inputs and fitness functions. (A) Fitness effects can be drawn from a gamma or normal distribution specified by the user. (B) Fitness effects may take the form of deep mutational scanning (DMS) substitution matrices for each protein, or (C) biophysical substitution matrices derived from computational tools. (D) The genotype-to-phenotype mapping is chosen by the user based on input. 

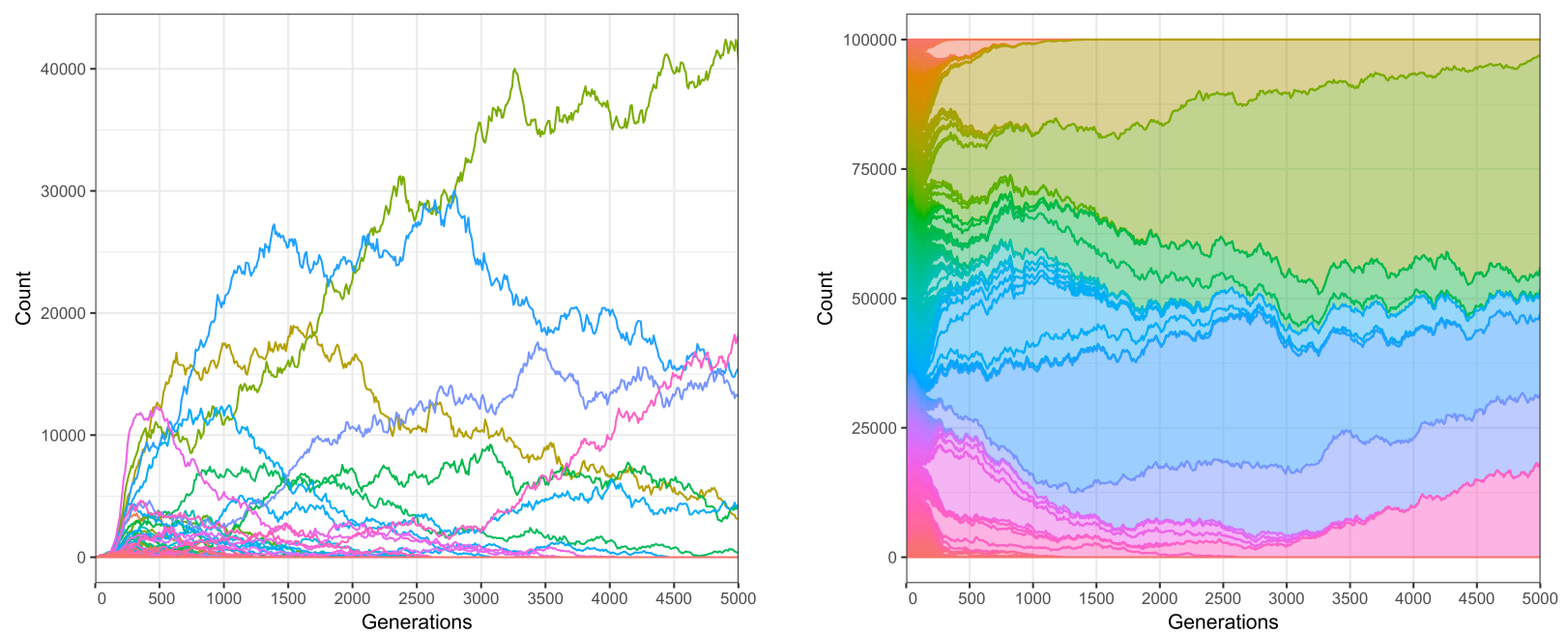

Figure 3. Test case 1: evolution under selection against misfolding toxicity. (A) SodaPop tracks the evolution of clones concurrently. Each color represents a single lineage identified by a barcode. The information in the left panel can also be represented as (B) the density of each lineage through time relative to the total population. Both these representations show pervasive clonal interference and competition.

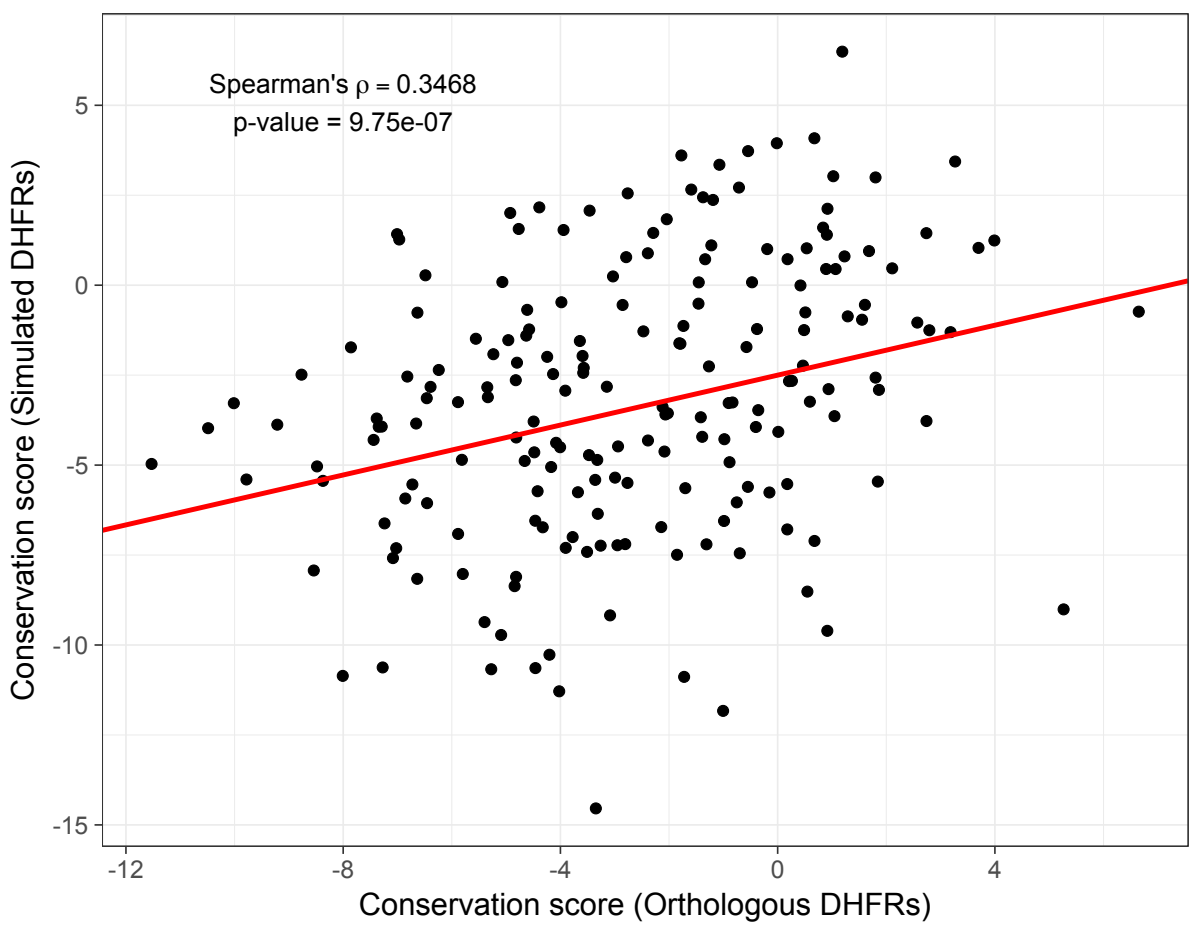

Figure 4. Test case 2: evolution under selection for thermodynamic stability. SodaPop captures a significant fraction of sequence conservation in DHFR. 


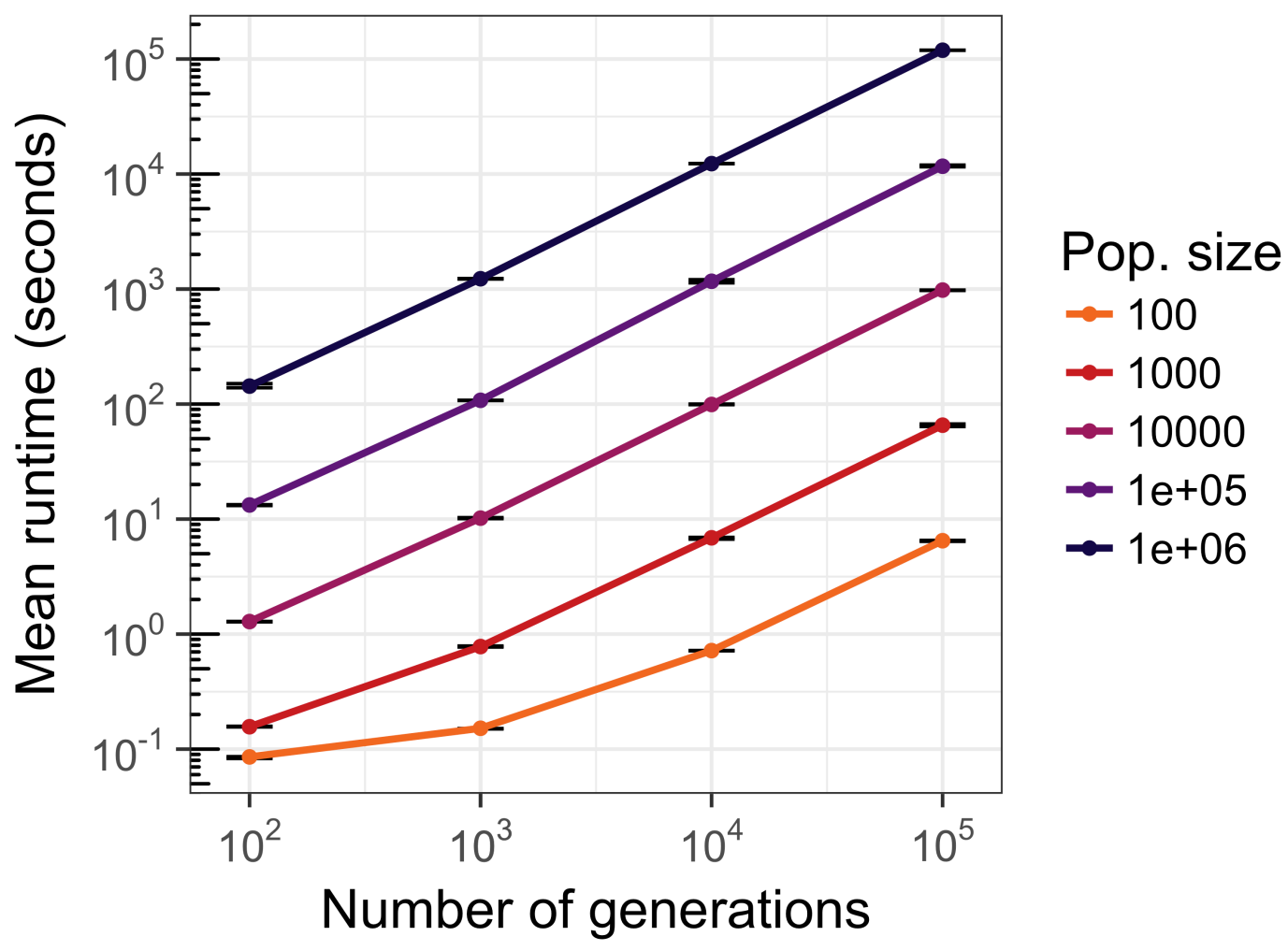

Figure 5. Benchmarking of SodaPop for different population sizes. Runtime of SodaPop with varying population 